\title{
Managing Cancer Care During the COVID-19 Pandemic: Agility and Collaboration Toward a Common Goal
}

\author{
Masumi Ueda, MD, MA ${ }^{1,2,3}$; Renato Martins, MD, MPH1,2,3; Paul C. Hendrie, MD, $\mathrm{PhD}^{1,2,4}$; \\ Terry McDonnell, DNP, ACNP-BC ${ }^{1}$; Jennie R. Crews, MD, MMM"1,3; Tracy L. Wong, MBA ${ }^{1}$; Brittany McCreery, MD, MBA ${ }^{1}$; \\ Barbara Jagels, RN, MHA ${ }^{1}$; Aaron Crane, MHA ${ }^{1}$; David R. Byrd, MD ${ }^{1,5}$; Steven A. Pergam, MD, MPH ${ }^{1,6,7}$; \\ Nancy E. Davidson, MD ${ }^{1,2,3}$; Catherine Liu, MD ${ }^{1,6,7, *}$; and F. Marc Stewart, MD ${ }^{1,2,3, *}$
}

\begin{abstract}
The first confirmed case of coronavirus disease 2019 (COVID-19) in the United States was reported on January 20,2020, in Snohomish County, Washington. At the epicenter of COVID-19 in the United States, the Seattle Cancer Care Alliance, Fred Hutchinson Cancer Research Center, and University of Washington are at the forefront of delivering care to patients with cancer during this public health crisis. This Special Feature highlights the unique circumstances and challenges of cancer treatment amidst this global pandemic, and the importance of organizational structure, preparation, agility, and a shared vision for continuing to provide cancer treatment to patients in the face of uncertainty and rapid change.
\end{abstract}

J Natl Compr Canc Netw 2020;18(4):366-369 doi: $10.6004 /$ jnccn.2020.7560

\footnotetext{
${ }^{1}$ Seattle Cancer Care Alliance; ${ }^{2}$ Clinical Research Division, Fred Hutchinson Cancer Research Center; ${ }^{3}$ Division of Medical Oncology, Department of Medicine, University of Washington; ${ }^{4}$ Division of Hematology, Department of Medicine, University of Washington; ${ }^{5}$ Department of Surgery, University of Washington; ${ }^{6}$ Vaccine and Infectious Diseases Division, Fred Hutchinson Cancer Research Center; and ${ }^{7}$ Division of Allergy and Infectious Diseases, Department of Medicine, University of Washington, Seattle, Washington
}

The first confirmed case of coronavirus disease 2019 (COVID-19) in the United States was reported on January 20, 2020, in Snohomish County, Washington. ${ }^{1}$ As of March 14, 2020, Washington state had 642 cases and 40 deaths. ${ }^{2}$ In the United States, we are fortunate to provide care for patients with cancer in a resource-rich environment with state-of-the-art, life-saving treatments. However, the reality of diminishing supplies and hospital resources in the face of the COVID-19 pandemic is a departure from our usual state. The potential threat of COVID-19 to our immunocompromised patients as a result of their disease or the treatment we provide is thought to be significant, tipping the usual risk/benefit balance in extreme ways. There are limited data on immunosuppressed hosts, but early published reports from China on the outcomes of patients with cancer infected with COVID-19 indicated a 3.5 times higher risk of needing mechanical ventilation or ICU admission or dying compared with patients without cancer. ${ }^{3}$ Although the case numbers in our patients at this point are low, ongoing community transmission essentially ensures this will not remain static. Due to the projected shortage of health care resources, as well as the predicted increased risk of cancer treatment during this time, difficult decisions about how and when to provide cancer treatment have become a necessity. The challenges we face as an institution, however, must still align with our overarching goal, which remains to provide cancer treatment to patients in need, as safely and as justly as possible.

\section{Initial Preparations}

The first step toward this goal is driven by robust infection and environmental control. Triage of patients with respiratory symptoms is critical to reduce exposure to other patients and staff. Early identification and masking of individuals with respiratory symptoms is routine during respiratory virus season, but this was initiated at entry points throughout the system where symptom screening 
of all patients, visitors, and staff took place before entering our outpatient clinics and hospital. In the clinic, symptomatic patients are diverted to a separate secondary screening area for consideration for COVID-19 testing. In Seattle, we are fortunate to have early access to COVID-19 testing, ${ }^{4}$ which was rapidly scaled up, allowing staff to perform nasopharyngeal swabs for symptomatic patients meeting COVID-19 testing criteria ${ }^{5}$ on entering the facility.

Diverse patient and family education for infection prevention are also needed. Patient handouts, signs, and website to provide COVID-19 education were quickly developed. To address questions, we established a phone triage line for patients with mild symptoms in the community to minimize exposures in our clinic and help patients avoid emergency departments. Testing at a drive-up site was coordinated when appropriate.

Reinforcement of a strict "stay at home when ill" policy and access to testing for symptomatic staff have been key to limiting exposures. A comprehensive policy for testing staff, tracking results for persons under investigation, tracing exposures, and defining return to work is essential to maintain a stable workforce. All policies and guidelines, including videos for proper donning and doffing of personal protective equipment (PPE), should be consolidated in one webpage for quick access., Other infection control measures include restriction of travel and enabling work-from-home by prioritizing information technology resources.

Concurrently with infection control, early coordination of institution-wide efforts is essential to manage rapidly changing information. Forming an "Incident Command" structure (Figure 1) is necessary to centralize all information given to staff, patients, and the community, and to consolidate and communicate the work of many individual groups. Virtual town halls with leadership are essential for clear and consistent messaging. Leveraging media resources for real-time patient communication is vital. Outside of our organization, shared portals with area health systems and community partners were created to coordinate efforts and offer support. During this early period, it is important to be flexible and ready for unexpected challenges. Leadership must identify system weaknesses, gaps in knowledge, and areas of concern. As basic crisis management, leadership, and strategies for

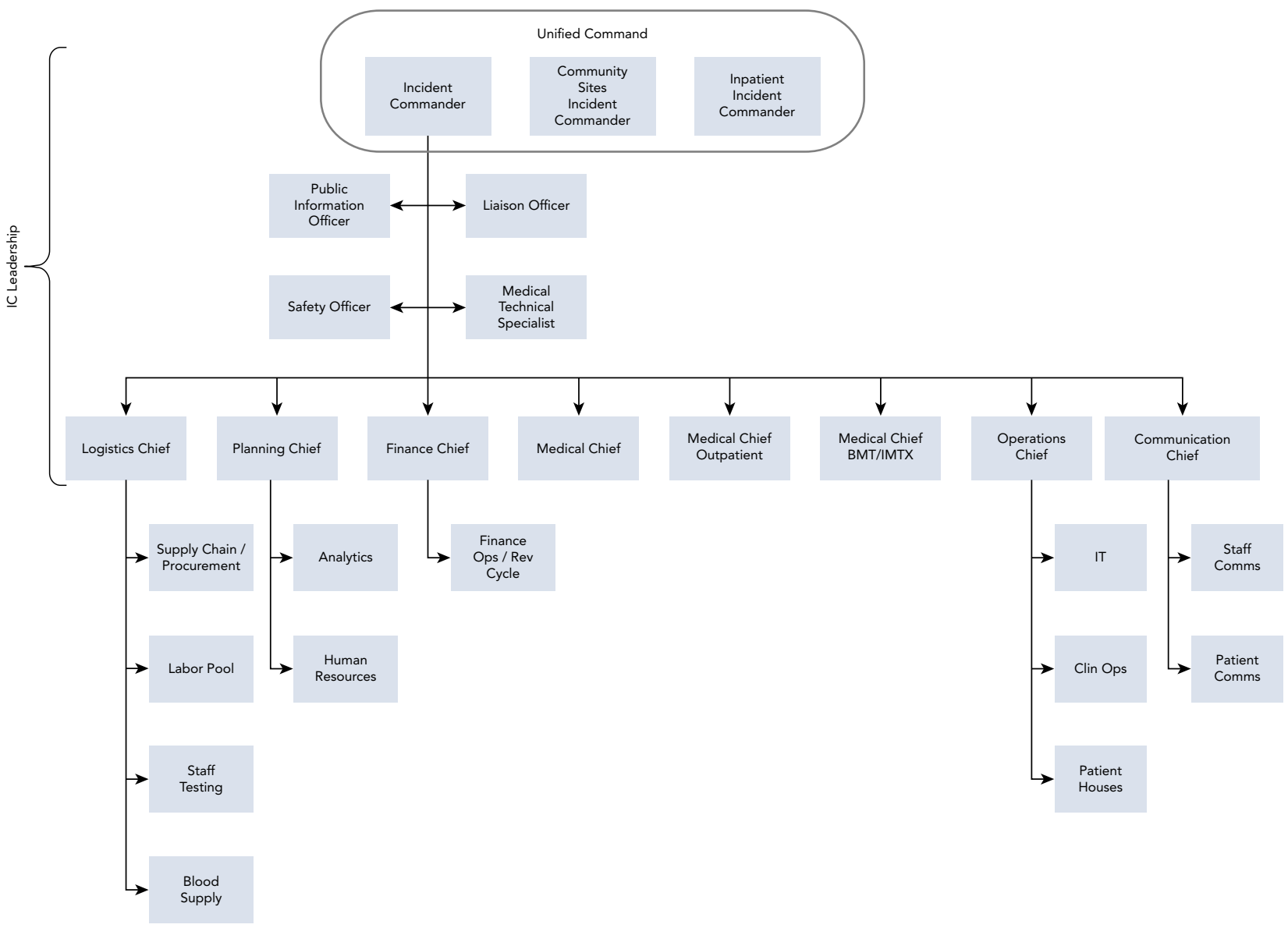

Figure 1. The Incident Command structure at Seattle Cancer Care Alliance.

Abbreviations: Comms, communications; IC, Incident Command; IT, information technology; Ops, operations. 
prevention and communication are established, it is important to then develop strategies for care continuity.

\section{Outpatient Considerations}

As a referral center, we see many patients from outside our region, and concerns for exposing non-local patients to COVID-19 grew as the virus spread. In addition, expected staff shortage due to school closings pose significant staffing limitations. One of our initial priorities was to establish a multilayer coverage system for the clinics in case providers had to be quarantined on short notice. Subsequently, medical and operational leadership determined that all "well" visits for existing patients would be rescheduled or transitioned to telemedicine. We have rapidly expanded telemedicine efforts through expedited physician credentialing, training, and modification based on changing regulations. Consultations for second opinions when patients are already under treatment in another system have been deferred. The center has also increased our hours of operations and acute evaluation capabilities to ensure that emergency departments and hospital resources are reserved for those absolutely requiring higher-level care.

\section{Treatment Decisions}

Such shifts in outpatient care delivery pose logistic challenges, but the decisions are relatively straightforward. The more difficult decisions are clinical decisions regarding delay of treatment for patients who are currently undergoing chemotherapy or about to begin. For solid tumor patients, adjuvant therapy with curative intent likely should proceed, despite the threat of COVID-19 infection during treatment. For patients with metastatic disease, treatment delays may lead to worsening performance status and loss of the window to treat. Considerations should include how such delays may lead to admission for symptom palliation, which further stresses inpatient resources. Though cancer surgery is not considered elective, surgical intervention also needs prioritization; there is currently a 2 -week ban on elective surgery in our system due to limited availability of PPE, staffing, and bed capacity. Changes to usual practice are necessary. For example, several months of endocrine therapy and delay in surgery may be appropriate for some patients with early-stage hormone receptor-positive breast cancer. Surgeon-to-patient phone calls have optimized shared decision-making to delay surgery.

Patients with aggressive hematologic malignancy have similar or greater urgency for life-saving treatment. Stem cell transplantation and cellular immunotherapies provide curative treatments for many with aggressive disease and in many cases cannot be delayed. Travel bans limit access to international donors for allogeneic stem cell transplantation, and cryopreservation of donor products has been recommended. ${ }^{7}$ Our oncology disease groups are tasked with determining what treatments can be selected to lessen immunosuppression, moved from the inpatient to outpatient clinic, ${ }^{8}$ and delayed.

Enrollment in clinical trials for all diseases will have to be limited to those that are most likely to benefit our patients. To what extent we can prioritize the therapies given to individual patients to reduce the burden on our system under stress is an ongoing challenge. Cancer centers should make it their mission to do all possible to continue to keep their doors open to provide care, unless there comes a time when staff and patient safety are no longer tenable.

\section{Ethical Considerations}

Beyond the care of the individual patient, oncology clinicians will face the heavy reality of rationing care. As the pandemic progresses, there will come a point when channeling a large amount of resources for an individual patient will be in direct conflict with the greater social good. If an oncology patient with late-stage disease or with comorbid health conditions such as heart or lung dysfunction acquires COVID-19 and requires mechanical ventilation, the prognosis is likely to be dismal. According to a recent retrospective study from Wuhan, China, only one patient survived among 32 who were seriously ill with confirmed COVID-19 and required mechanical ventilation. ${ }^{9}$ Thus, we believe it is imperative to have proactive end-of-life and palliative care discussions with patients with cancer who may become infected with COVID-19. Although these practices should be a part of routine oncology care, such discussions with all patients with cancer has become even more vital in these times. It is our duty to not only educate but also provide resources to help patients make decisions regarding treatment during this period of uncertainty. With dwindling resources, oncologists must also consider carefully what treatments are most likely to be successful, symptom-relieving, or lifesaving, and consider those patients likely to get the greatest benefit from treatments. Proactive discussions surrounding these challenging decisions should occur among disease-specific groups, medical ethicists, and palliative care teams.

\section{Hospital Management of Oncology Patients}

The priority for inpatient oncology units has been to prepare for upcoming shortages of beds and resources due to the predicted surge of patients with COVID-19 requiring acute care and ICU beds. Despite some centers having beds or facilities dedicated to cancer care, large outbreaks may require reallocation of units, hospital wards, or even entire systems to care for patients during pandemics. For both the clinic and the hospital, blood product shortages due to decline in community blood drives necessitate stricter adherence to and considering lower thresholds for transfusion. ${ }^{5}$ Transferring possibly infected patients from the clinic to the hospital requires logistic planning to reduce exposure to staff and public. Due to shortages from 
increased consumption, creative solutions to conserve PPE must be developed, including favoring soap and water hand washing over hand gel use in standard precaution rooms, limiting the number of team members entering patient rooms, and reducing PPE-requiring nursing procedures, such as measurement of urine outputs.

To protect staff, training sessions for PPE were updated and made available on a daily and hourly basis. To protect the public and patients from exposures and to reduce PPE consumption, we adopted a no visitor policy in our inpatient units with rare exceptions such as for end-of-life circumstances. These decisions, made in conjunction with patient advisors, were difficult for both our patients and families and medical staff who recognize the important contributions of family/friend support for our hospitalized patients with cancer.

\section{Employee and Leadership Well-Being}

Finally, the emotional and physical well-being of our staff and faculty requires proactive attention. Provider burnout is expected, and priority should be given to protect the health of frontline staff and to assure a safe work environment. Additionally, attention is needed to human resources for policy and compensation development for furloughs and mandatory isolation, and the creation of a back-up labor pool. Reassignment of clinical duties to administrative roles should be considered for staff who are immunocompromised or have significant comorbidities that put them at increased risk with COVID-19. Finally, we have made a conscious effort to recognize the difficult job we are all doing and the importance of self-care. In fact, the leadership of our Incident Command shifts every 2 weeks by design to assure needed downtime for rest.

\section{Looking Forward}

The COVID-19 pandemic has presented unique challenges and learning opportunities for cancer centers. The future trajectory of this pandemic is uncertain, and we must continue to prepare for its widespread impact. The situation is dynamic, and policies and recommendations may change at any time. As we write, the healthcare crisis surrounding COVID-19 continues to evolve, and new conditions could change some of our existing recommendations. We expect to get through these uncertain times, but the long-term financial and emotional downstream impacts will be future challenges. The overarching goal, however, is to continue to provide compassionate, safe care for patients with cancer. We hope to continue to serve as a resource for our patients and to the cancer community as this pandemic continues to spread across the United States. To many of us, this has become the health care challenge of our generation, one that modern cancer therapy has never had to face. We will prevail, and when the pandemic ends, we will all be proud of what we did for our patients and each other in this critical moment for humanity.

\section{Acknowledgments}

The authors would like to thank all staff on the front lines at the Seattle Cancer Care Alliance, Fred Hutchinson Cancer Research Center, and University of Washington who have been working tirelessly to ensure that patient care continues safely during this public health emergency. We would like to thank all of our colleagues at these institutions in Infectious Disease, Blood and Marrow Transplantation and Immunotherapy, Hematologic Malignancies, Solid Tumor disease groups, Pulmonary and Critical Care, Surgical Oncology, Radiation Oncology, Transfusion Medicine, Laboratory Medicine, Palliative Care, and Social Work who have been working countless hours developing specialty-specific guidelines regarding COVID-19 on a local and national level. Finally, our commitment to patients would not be possible without the leaders and staff in Infection Prevention, Facilities, Quality Improvement, Information Technology, and many others who continue to contribute enormous efforts in our institution's response during the COVID-19 pandemic.

Submitted March 15, 2020; accepted for publication March 16, 2020

Disclosures: The authors have disclosed that they have no financial interests, arrangements, affiliations, or commercial interests with the manufacturers of any products discussed in this article or their competitors.

Correspondence: Masumi Ueda, MD, MA, Clinical Research Division, Fred Hutchinson Cancer Research Center, 1100 Fairview Avenue N, D1-100, Seattle, WA 98109. Email: mueda@fredhutch.org

\section{References}

1. Holshue ML, DeBolt C, Lindquist S, et al. First case of 2019 novel coronavirus in the United States. N Engl J Med 2020;382:929-936.

2. 2019 Novel Coronavirus Outbreak (COVID-19): Washington State Department of Health. Available at: https://www.doh. wa.gov/Emergencies/Coronavirus. Accessed March 16, 2020

3. Liang W, Guan W, Chen R, et al. Cancer patients in SARS-CoV-2 infection: a nationwide analysis in China. Lancet Oncol 2020;21:335-337.

4. SARS-CoV-2 (COVID-19) Qualitative PCR. Available at: https:// testguide.labmed.uw.edu/public/view/NCVQLT. Accessed March 16, 2020.

5. COVID-19 Response Tools: Seattle Cancer Care Alliance. Available at: https://www.seattlecca.org/covid-19-screening-tools. Accessed March 16, 2020

6. UW Medicine COVID-19 Resource Site. Available at: https://covid-19. uwmedicine.org/Pages/default.aspx. Accessed March 16, 2020.

7. NMDP/Be The Match Response to COVID-19. Available at: https:// network.bethematchclinical.org/news/nmdp/be-the-match-response-tocovid-19/. Accessed March 16, 2020.

8. National Comprehensive Cancer Network (NCCN) Best Practices Committee Infusion Efficiency Workgroup Toolkit: Providing Oncology Treatments in the Outpatient Setting. Available at: https://www.nccn.org/ about/news/ebulletin/ebulletindetail.aspx?ebulletinid=3745. Accessed March 16, 2020.

9. Zhou F, Yu T, Du R, et al. Clinical course and risk factors for mortality of adult inpatients with COVID-19 in Wuhan, China: a retrospective cohort study [published March 11, 2020]. Lancet, doi: 10.1016/S0140-6736(20)30566-3 